\title{
Launch Vehicle Manual Steering with Adaptive Augmenting Control: In-Flight Evaluations of Adverse Interactions Using a Piloted Aircraft
}

\author{
Curt Hanson ${ }^{1}$, Chris Miller ${ }^{2}$ \\ NASA Armstrong Flight Research Center, Edwards, CA, 93523 \\ John H. Wall ${ }^{3}$ \\ Dynamic Concepts, Inc., Huntsville, AL, 35806 \\ Tannen S. VanZwieten ${ }^{4}$, Eric T. Gilligan ${ }^{5}$ \\ NASA Marshall Space Flight Center, Huntsville, AL, 35812 \\ Jeb S. Orr ${ }^{6}$ \\ The Charles Stark Draper Laboratory, Inc., Huntsville, AL, 35806
}

\begin{abstract}
An Adaptive Augmenting Control (AAC) algorithm for the Space Launch System (SLS) has been developed at the Marshall Space Flight Center (MSFC) as part of the launch vehicle's baseline flight control system. A prototype version of the SLS flight control software was hosted on a piloted aircraft at the Armstrong Flight Research Center to demonstrate the adaptive controller on a full-scale realistic application in a relevant flight environment. Concerns regarding adverse interactions between the adaptive controller and a potential manual steering mode were also investigated by giving the pilot trajectory deviation cues and pitch rate command authority, which is the subject of this paper. Two NASA research pilots flew a total of 25 constant pitch rate trajectories using a prototype manual steering mode with and without adaptive control, evaluating six different nominal and off-nominal test case scenarios. Pilot comments and PIO ratings were given following each trajectory and correlated with aircraft state data and internal controller signals post-flight.
\end{abstract}

\section{Nomenclature}

AAC $=$ Adaptive Augmenting Control

AFRC $=$ Armstrong Flight Research Center

ARI $=$ attitude reference indicator

DDI $=$ digital display interface

FAST $=$ Full-Scale Advanced Systems Testbed

GCD $=$ Game Changing Development

HUD $=$ heads up display

ILS $\quad=$ instrument landing system

$\mathrm{LOV}=$ loss of vehicle

LVAC $=$ Launch Vehicle Adaptive Controls

MSFC $=$ Marshall Space Flight Center

MSM $=$ manual steering mode

${ }^{1}$ Aerospace Engineer, Controls and Dynamics Branch, MS 4840D, AIAA Nonmember.

${ }^{2}$ FAST Chief Engineer, Systems Engineering and Integration Branch, MS 4840D, AIAA Nonmember.

${ }^{3}$ SLS Flight Controls Lead, Guidance, Navigation and Control Branch, MS EV40, AIAA Member.

${ }^{4}$ Associate Principal Engineer, NASA Engineering and Safety Center, MS EE04L, AIAA Member.

${ }^{5}$ Aerospace Engineer, Control Systems and Analysis Branch, MS EV41, AIAA Nonmember.

${ }^{6}$ Senior Member of the Technical Staff, Dynamic Systems and Control, MS EV41, AIAA Nonmember. 


$\begin{array}{ll}\text { NDI } & =\text { nonlinear dynamic inversion } \\ \text { NESC } & =\text { NASA Engineering and Safety Center } \\ \text { OCT } & =\text { Office of the Chief Technologist } \\ \text { PDR } & =\text { preliminary design review } \\ \text { PID } & =\text { proportional plus integral plus derivative } \\ \text { PIO } & =\text { pilot in the loop oscillation } \\ \text { SLS } & =\text { Space Launch System } \\ \text { SRB } & =\text { Solid Rocket Booster } \\ e_{r} & =\text { pitch rate tracking error } \\ J_{A} & =\text { measure of pilot aggressiveness } \\ k_{T} & =\text { adaptive gain } \\ q_{c m d} & =\text { pitch rate command from pilot } \\ \bar{q}_{c m d} & =\text { steady-state component of pitch rate command } \\ \dot{q}_{c m d} & =\text { pitch acceleration command } \\ q_{e r r} & =\text { pitch rate guidance error } \\ q_{f b k} & =\text { pitch rate feedback } \\ t_{0} & =\text { initial time } \\ t_{f} & =\text { final time } \\ y_{s} & =\text { power measurement of parasitic dynamics } \\ \tau & =\text { time index } \\ \Delta \tau & =\text { elapsed time between data points }\end{array}$

\section{Introduction}

$\mathrm{T}$ he Space Launch System (SLS) Block I vehicle (Fig. 1) will deliver more payload to orbit and produce more thrust than any other foreign or domestic launch vehicle, opening the way to new frontiers of space exploration. Like all large launch vehicles, SLS must balance the competing needs of maximizing performance while maintaining robustness. With its high thrust, large size, and multiple elements, SLS is a highly flexible structure with complex bending characteristics. Control commands must be allocated to each of its six engines, which are actuated along the pitch and yaw axes by thrust vector control actuators with limited bandwidth. The propellant tanks have lightly damped lateral sloshing modes, and the large payload envelope produces uncertain parasitic dynamics. The SLS trajectories are optimized to maximize performance, which further challenges the flight control design and leaves little margin to share among multiple disciplines and subsystems. ${ }^{1}$

Development of an Adaptive Augmenting Control (AAC) algorithm, as an addition to the classical control architecture for launch vehicles, began at NASA MSFC during the Constellation Program. Development of the AAC was accelerated during the SLS Program. Maturation of the algorithm, coupled with the simulation demonstration of improved performance and robustness, led to the AAC algorithm being baselined as part of the autopilot design and flight software build prior to the SLS Preliminary Design Review (PDR) in 2013. ${ }^{2}$ Leading into the PDR, the AAC remained the only component of the SLS control system that had not yet been flight tested; all other dynamic elements were successfully tested through Ares I-X. ${ }^{3}$ Demonstration of the AAC algorithm in a relevant environment was achieved in late 2013 during the Launch Vehicle Adaptive Controls (LVAC) experiment, an implementation and flight evaluation of the prototype SLS control system software in the Full-Scale Advanced Systems Testbed (FAST) (Fig. 2) at the NASA Armstrong Flight Research Center (AFRC). ${ }^{4,5}$

The LVAC experiment was a partnership between the NASA MSFC, the NASA AFRC, the NASA Engineering and Safety Center (NESC) and the NASA Office of the Chief Technologist (OCT) Game Changing Development (GCD) Program. There were three primary objectives for the SLS control system evaluation on the FAST aircraft:

1. Demonstrate closed-loop tracking with negligible adaptation in an environment that is commensurate with the nominal controller design envelope.

2. Demonstrate improved performance in an environment where the nominal controller performance is less than desired.

3. Demonstrate the ability to recover from unstable, mis-modeled parasitic dynamics to a bounded nondestructive limit cycle.

One element of the SLS control system still under consideration at the time of the PDR was a human-in-the-loop manual steering mode (MSM). Manual steering allows the crew to have direct, on-board control of the SLS trajectory through the flight control system after separation of the Solid Rocket Boosters (SRBs). Prior flight experience with adaptive control of aerospace vehicles has shown the potential for adverse interactions between the pilot and the 
adaptive controller. ${ }^{6-8}$ Experimental characterization of this interaction with critical analysis from pilot evaluations may assist in determining whether the AAC should remain active if manual steering is engaged, in the event that the SLS Program decides to employ a MSM for the operational vehicle. For this reason, an additional objective was added to the LVAC flight experiment.

4. Explore interactions between manual steering and the AAC.

No official SLS manual steering design existed prior to the flight experiment, so the test team implemented a prototype design based on system requirements informally-derived from previous rocket manual steering designs. This paper describes the AAC algorithm, the prototype design for the SLS manual steering mode, and a suite of test scenarios representing a wide variety of off-nominal launch events. Flight results of the manual steering mode with and without the AAC active are presented. Possible adverse interactions between the AAC and MSM are discussed and recommendations given for consideration in the final SLS manual steering mode design.

\section{Approach}

\section{A. AAC Overview}

The three summary-level objectives of the AAC are as follows:

1. "Do no harm;" return to baseline control design when not needed.

2. Increase performance; respond to error in ability of vehicle to track commands.

3. Regain stability; respond to undesirable control-structure interaction or other parasitic dynamics.

The result is a decrease in the likelihood of loss of mission/vehicle or an extension in the abort eligibility in the event of an anomaly (e.g., case breach). A unique feature of the algorithm is its capability to protect against adverse control structure interactions.

The AAC is designed to maintain nominal system performance, exhibit a predictable response, and to be compatible with classical stability criteria by augmenting the baseline autopilot system. Within the design envelope of the vehicle, the adaptive system maintains the nominal control system gains. For conditions outside the design envelope, the AAC provides additional robustness by adjusting the forward-loop gain to recover from poor closed-loop performance and prevent or delay loss of vehicle. The adaptive update law increases the gain when excessive tracking error indicates that system performance is less than preflight predictions. The gain is decreased when excessive power within a prescribed frequency band is observed in the control command as a result of control coupling with launch vehicle parasitic dynamics, such as flexibility or fuel slosh.

Figure 3 shows a simplified block diagram of the SLS pitch control system with the PDR version of the adaptive augmenting control algorithm. ${ }^{1}$ The adaptive update law increases the adaptive feed-forward gain $k_{T}$ in response to pitch rate tracking error $e_{r}$ and decreases $k_{T}$ for undesirable resonance in the control loop due to parasitic dynamics. A spectral damper computes undesirable resonance by squaring the output of a high-pass filter on the difference between a reference model and the control signal, then passing the result through a low-pass filter. This provides a measure of the energy of the parasitic dynamics within a prescribed frequency band of interest.

The adaptive gain $k_{T}$ is multiplicative on the output of the baseline proportional plus integral plus derivative (PID) controller, balancing attitude tracking with the attenuation of undesired frequency content within the control path. The gain is constrained to stay within the baseline controller's design margin of $\pm 6 \mathrm{~dB}$, or a minimum value of 0.5 and a maximum value of 2 .

The algorithm was designed under the assumption that the gain-scheduled attitude control design is well-tuned for a nominal vehicle and trajectory at each flight time, meaning that adaptation should only occur should the vehicle performance lie outside the nominal design envelope. The adaptive law, therefore, also contains a leakage term designed to attract the adaptive gain to unity, so that only small alterations are made to the fixed-gain design within the expected dispersion envelope, without requiring the use of a dead zone in the update law. Additional description of the AAC algorithm can be found in Refs. 1 and 2.

\section{B. SLS Control Software Integration on the Test Aircraft}

The FAST aircraft is a specially modified single-seat F/A-18 aircraft with extensive instrumentation and a research flight control system. The prototype SLS controller and autopilot software, with only the pitch channel activated and the disturbance compensation algorithm disabled, was hosted in the research flight control system, along with a simulation of the SLS vehicle that included rigid body dynamics, structural and slosh dynamics, atmospheric disturbances, and a variety of failure modes. This simulation served as a reference model for a nonlinear dynamic inversion (NDI) controller, which suppressed the underlying F/A-18 dynamics and replaced them with SLS dynamics. Thrust vector pitch commands from the SLS controller, fuel slosh dynamics and atmospheric disturbances were allocated to the symmetric stabilators. Symmetric ailerons were used to generate flexible mode pitch dynamics from 
the SLS simulation. Under NDI control, the FAST aircraft exhibited a high degree of dynamic similarity to the SLS vehicle, closely matching time- and frequency-domain responses in pitch angle error, pitch rate, pitch acceleration and variations in angle of attack. ${ }^{4,5}$

The aircraft's trajectory was designed to start at approximately 35 deg nose-high and track a nose-down pitch rate guidance command of approximately $0.75 \mathrm{deg} / \mathrm{s}$, which is near the average pitch rate of the SLS during its gravity turn prior to separation of the vehicle's SRBs. The experiment was not designed to match the aircraft's pitch attitude to that of SLS, since this would require a near-vertical flight path, which is not achievable with an aircraft thrust-to-weight ratio of less than unity. Pilot control of the throttles consisted of full military (non after-burner) power during nose-high conditions, followed by a gradual reduction to flight idle as the pitch attitude passed through horizontal. This approach maximized the duration of the trajectory. Total trajectory test time was approximately 70 seconds, which represents a sufficiently large portion of the SLS boost trajectory to allow a thorough evaluation of the controller's performance, including adaptation to unexpected events.

Prior to each trajectory, the pilot configured the experiment using the digital display interface (DDI). Entries to the DDI allowed the pilot to select specific test cases that represented nominal and off-nominal scenarios, such as unmodeled flexible dynamics or thrust vector system failures. At any time during the trajectory, the pilot could disengage the SLS guidance command and engage the manual steering mode via the nose wheel steering button on the control stick.

The onboard SLS reference model was also used to estimate conditions that would lead to a loss of vehicle (LOV) for the SLS rocket. The experiment monitored these estimates and disengaged the research flight control system for exceeding pre-determined limits for either rigid body loads, or vehicle flexure. Rigid body loads were considered to be proportional to dynamic pressure and angle of attack, typically referred to as q-alpha. For the purposes of the experiment, structural failure was assumed to occur if the simulated vehicle tip deflection exceeded $5 \mathrm{ft}$ or the rigid body load indicator exceeded 8,500 deg-lbf/sq ft.

\section{Manual Steering Mode Implementation}

Pilot control introduces an additional dimension of uncertainty into the robustness analysis of aerospace vehicles, given the ability of the pilot to adjust the closed-loop gain of his or her dynamic response; ${ }^{9}$ this is especially true for adaptive systems, such as the AAC, that also adjust the system gain. When both pilot and adaptive system make gain adjustments based upon the same feedback parameters, such as tracking error, the potential exists for adverse interactions between them.

Experimental characterization of this interaction, including pilot evaluations, may assist in determining whether the AAC should remain active if manual steering is engaged, in the event that the SLS Program decides to employ a MSM for the operational vehicle. No official SLS manual steering design existed at the time of the flight experiment, so the test team implemented a prototype design based on system requirements informally-derived from previous rocket manual steering designs. It should be noted that the control stick characteristics and out-the-window view, among other factors, differ between the LVAC experiment and the eventual SLS crew environment.

Pilot longitudinal stick commands were linearly scaled to pitch rate guidance commands at 1 degree per second per inch. The system provided a total command authority of \pm 2 in, or \pm 2 deg per second. The pilot lateral stick and rudder pedals were disabled with the SLS control laws engaged. Lateral-directional control was maintained by a separate autopilot.

The instrument landing system (ILS) glideslope deviation needle on the attitude reference indicator (ARI) displayed pitch attitude error to the pilot. As shown in Fig. 3, the ARI position at the top of the instrument panel, near the heads-up display (HUD), allowed the pilot to maintain out the window situational awareness. The linear display range of the ARI was \pm 2.5 deg of pitch attitude error.

The pilot control input is a rate command. The command is integrated and both rate and attitude errors are computed as inputs into the control laws. The trajectory error cue displayed to the pilot was based on a fixed $0.75 \mathrm{deg} / \mathrm{s}$ nose-down command, the same as the SLS guidance command for the autopilot (non-manual steering) test points.

\section{Evaluation Metrics}

Several metrics were used to evaluate the degree of interaction, whether beneficial or adverse, between the pilot and the adaptive control system.

\section{PIO Rating Scale}

A pilot in the loop oscillation (PIO) is an instability resulting from efforts by the pilot to control the vehicle. A PIO is characterized by pilot control inputs that are 180 deg out of phase with the response of the controlled error state of 
the vehicle. Pilot in the loop oscillation tendencies in a system are often revealed following abrupt or aggressive inputs. Figure 4 shows a rating scale from Ref. 10 used to quantitatively categorize the PIO tendency of an aircraft.

\section{Cumulative Tracking Error}

The trajectory tracking performance of the autopilot or the pilot using manual steering is measured quantitatively by plotting the integral of the square of the pitch attitude tracking error against time.

\section{Pilot Workload Metrics}

Pilot workload can be characterized by two measures: aggressiveness and duty cycle. ${ }^{11}$ Cross-plots of these measures for different configurations help identify changes in pilot workload.

Pilot aggressiveness $\left(J_{A}\right)$ is a measure of dynamic control deflection, calculated using Eq. (1) as the time-averaged summation of the unbiased pilot attitude rate command inputs during the trajectory. The command bias, $\bar{q}_{c m d}(\tau)$ is found by forward and backward low-pass filtering of the original signal. Filtering the data in both directions eliminates any filter-induced phase shift. Removal of the command bias avoids the errant categorization of steady, non-zero pilot inputs as highly aggressive commands. The unbiased control measure is then normalized by the total allowable command range.

$$
J_{A}=\frac{100 \%}{t_{f}-t_{0}} \sum_{\tau=t_{0}}^{t_{f}}\left(\frac{\left|q_{c m d}(\tau)-\bar{q}_{c m d}(\tau)\right|}{q_{c m d}^{\max }-q_{c m d}^{\min }}\right) \Delta \tau
$$

Pilot duty cycle is a measure of how frequently the pilot makes significant control adjustments. Duty cycle is calculated by counting the number of peaks per second in the command input signal. A peak is arbitrarily defined as a change in the signal magnitude of at least 10 percent of the command's total allowable range and in the direction opposite of the signal's last previously identified change. It is a determination of the frequency with which the pilot reverses control direction.

\section{E. Test Approach}

In total, the LVAC experiment gathered data for 25 test trajectories flown under manual steering mode, 13 with Pilot A on flight 143, and 12 with Pilot B on flight 144. The 25 test trajectories consisted of 6 different test cases covering the 3 main objectives of the program. ${ }^{5}$ Test cases $5,7,15,16$, and 17 represent extreme events that are well outside the design envelope of the SLS. Table 1 lists the manual steering mode test matrix.

Table 1. Manual steering mode test matrix.

\begin{tabular}{cclcccc}
\hline Objective & Case & SLS scenario description & Card & AAC & $\begin{array}{c}\text { Pilot A } \\
\text { (number of attempts) }\end{array}$ \\
\hline 1 & 0 & Nominal Plant and Environment & 5 & on & 2 & 2 \\
& & & 6 & off & 2 & 2 \\
\hline 2 & 5 & Two Spaced Hard-over Failures & 7 & off & 1 & 1 \\
& \multirow{2}{*}{7} & & 8 & on & 1 & 1 \\
& & Wind Shear with Two Hard-over & 11 & off & 1 & 1 \\
& \multirow{2}{*}{15} & Failures & 12 & on & 2 & 1 \\
\hline \multirow{2}{*}{3} & \multirow{2}{*}{16} & High Gain with Slosh Excitation & 25 & off & 0 & 1 \\
& \multirow{2}{*}{17} & High Gain with Unstable Flex & 26 & on & 0 & 1 \\
& & & 19 & off & 1 & 0 \\
& & & 20 & on & 1 & 0 \\
& & & 29 & off & 1 & 1 \\
& & & 30 & on & 1 & 1 \\
\hline
\end{tabular}

In most instances, both pilots evaluated a given test case to account for differences in pilot technique and rating methodology. Back-to-back evaluations with and without adaptation helped to reduce uncertainty due to variations in atmospheric effects and aircraft mass properties to the greatest extent possible. The 'Nominal Plant and Environment' 
test case was evaluated at the beginning and at the end of each flight to establish a trend for pilot adaptation to the task.

Test pilots rated each configuration using the aircraft PIO rating scale. Ratings were given immediately following the completion of each test point. The aircraft hot mic system transmitted comments made by the pilot during the test point to the ground control room for situational awareness. This audio stream was also recorded along with HUD video for post-flight analysis.

\section{Results}

Figure 5 presents the pilot PIO ratings for the 25 test cases flown under manual steering mode. For some test points, Pilot B provided separate ratings for the gross acquisition (initial capture) and fine tracking portions of the task.

An 'attempt' is defined as a pair of test cards, AAC-off and AAC-on, for a single test case. A comparison of the ratings between the first and second attempts for Case 0 shows that both pilots appear to have altered their piloting technique during the flights. For example, Pilot A rated the AAC-off configuration as having divergent oscillations (PIO 5) during the first attempt, but upgraded his assessment to undesirable motions (PIO 3) for the second attempt. An explanation can be found in the comments given with the ratings for these test points:

\section{Pilot A / Test Case 0 / AAC-off}

1st attempt - "Any attempt to tighten control leads to PIO. [...] Task performance is affected, but with a lot of compensation I can make this work."

2nd attempt - "Tight control definitely causes oscillations [...] they're not necessarily divergent $[\ldots]$ somewhat open-loop task [...] I'm going to call it a 3, although it's partially the way I flew it."

On the first attempt, Pilot A applied considerable compensation to maintain tracking performance. By the end of the flight, he had changed his piloting technique to use minimal compensation. The following comment from Pilot A further describes the altered technique:

\section{Pilot A / Test Case 5 / AAC-on}

"The technique for doing this is to not to try to fight the error but simply to set up a pitch rate that tracks it... it's almost an open-loop task."

Pilot technique should not be confused with pilot workload, although the former definitely has an effect on the latter. Technique refers to the degree to which the pilot enters the loop in an attempt to control the tracking error; it is the control strategy. Pilot workload is the measure of effort required to carry out the control strategy and achieve the desired level of tracking performance. Due to the evolution in pilot technique, comparisons of ratings between test cases, particularly those involving test cases at the beginning of the flight, may be misleading. An assumption is made in the following discussions that pilot technique did not vary significantly between the back-to-back AAC-off vs. AAC-on maneuvers for a given test case. A review of the recorded comments indicates that both pilots adjusted their techniques early in the flight and maintained a consistent control strategy thereafter.

\section{A. Objective 1: Minimal Adaptation in the Nominal Case}

The objective of Test Case 0: Nominal Plant and Environment is to show that the adaptive control gain stays near its initial value of one throughout the trajectory under both autopilot and manual control, indicating that no adaptation is taking place. Figure 6 shows the quantitative change in pilot workload between the AAC-off vs. AAC-on configurations for the 'Nominal Plant and Environment' test case. When two PIO ratings are given for a single point, the first is an assessment of the gross acquisition element of the task and the second is for fine tracking. Note that the reduced workload for Pilot A between the first and second attempts with AAC disabled matches the pilot comments discussed above.

In three of the four attempts for Test Case 0, adaptation resulted in equal or worse PIO ratings and higher pilot workload, as measured by both pilot aggressiveness and duty cycle. The largest increase in PIO rating and workload between AAC-off and AAC-on occurred during the second evaluation attempt by Pilot A. Figure 7 shows that for this attempt, tracking error also increased significantly during the AAC-on trajectory, as compared to both the AAC-off trajectory and an earlier AAC-on trajectory for the same test case flown under autopilot control. In fact, for all three attempts in which AAC-on produced worse ratings and higher workload, cumulative tracking error was higher than for the corresponding AAC-off trajectory. These trajectories also displayed a lower adaptive gain value than for the same test case as flown by the autopilot, as shown in Fig. 7. The lower gain values are a result of the pilot inputs, as discussed below.

The second attempt by Pilot B was unique among the Test Case 0 results in that the measurable pilot workload was lower with AAC activated as compared to the AAC-off trajectory. The PIO ratings given were slightly higher for 
AAC-on, primarily due to the presence of undesirable motions. As described below in the comments from Pilot B following the test, the workload was higher in terms of the concentration required, although that did not translate to measureable workload in terms of command inputs.

Pilot B / Test Case 0 / AAC-on

2nd attempt - "Gross acquisition, there was some undesirable motions - it affected the task [...] I had to concentrate on it more, so I would probably give it a 3. For the actual tracking task, probably a 2 - it did affect the task. [...] I had to concentrate a little bit more, and then as time went on it probably moved more like a 1 , but, there were some undes... nah, I would say no, because there were some undesirable motions. I'd just keep it a 2."

Figure 7 shows that the tracking error was much lower on the second attempt with AAC enabled by Pilot B than for the other three attempts. The adaptive gain also remained much closer to the value achieved under autopilot control, near the expected value of one for this test case.

Figure 8 shows the pilot pitch rate commands and spectral damper contributions to the adaptive gain for the second attempts by Pilot A and Pilot B. The increased workload of Pilot A is evident in both the magnitude and frequency of the command, as compared to that of Pilot B. Furthermore, adaptation due to the output of the spectral damper is much higher for Pilot A. Note that positive values of the spectral damper contribution result in a lower adaptive gain.

\section{B. Objective 2: Improved Tracking Performance}

Two test cases were flown to evaluate manual steering mode under Objective 2:

1. Test Case 5: Two Spaced Hard-over Failures, and

2. Test Case 7: Wind Shear with Two Simultaneous Hard-over Failures.

Each test case was flown by both Pilot A and Pilot B, with Pilot A flying the AAC-on test point for Test Case 7 twice. The primary metric for Objective 2, tracking performance, is shown in Fig. 9 for both test cases, and both pilots. For Test Case 5, the tracking error is equivalent between the automatically and manually flown points when AAC is off. Activation of the adaptive controller improved performance of the autopilot but resulted in larger tracking error under manual steering control.

Figure 10 shows the pilot workload metric for the Objective 2 manual steering mode trajectories. In all cases, the measured pilot duty cycle decreased when the adaptive controller was in the loop. Conversely, in all cases but one, activation of the adaptive controller resulted in equal or greater pilot aggressiveness. Pilot in the loop oscillation ratings for AAC-on were the same or slightly worse than for the AAC-off configuration, with the lone exception of Pilot B's assessment of fine tracking for Test Case 5. An explanation for the higher tracking error under manual steering with AAC enabled can be found by examining the two attempts by Pilot A for Test Case 7.

Pilot A flew Test Case 7 twice with AAC activated. Those test points were conducted back-to-back following the corresponding AAC-off test point, and showed significantly different workload characteristics. The first attempt had the highest measured pilot aggressiveness of all Objective 2 manual steering mode test points, while the second attempt had the lowest. The change in aggressiveness between the two attempts is due to a change in pilot technique, as described in the pilot comments:

Pilot A / Test Case 7 / AAC-on

1st attempt - "Getting into an oscillation. Seems divergent. Whether I can fix it or not, I don't know. Okay, there we go, I seem to have recovered somewhat. Don't know whether I can keep at it or not. Come on. Any real attempt to do the task leads to pretty good oscillations that seem divergent. [...] That was divergent, call it 5. A good, solid five."

2nd attempt - "If I'm really careful, I can sort of track this. It's very sensitive. [...] Okay, well in that case I'd give it a 4, but that's because I changed my piloting technique a lot and didn't really attempt tight control."

The pilot commands and associated adaptive gain values for the two AAC-on attempts by Pilot A at Test Case 7 are shown in Fig. 11. As observed in the Objective 1 manual steering mode results, high workload pilot commands are picked up by the spectral damper and tend to drive the adaptive gain toward its minimum value of 0.5 . In Test Case 7, the ideal response of the adaptive gain is to remain near 1 until the failure occurs (around 45 seconds), and then increase toward its maximum value of 2 . On the first attempt by Pilot A, the adaptive gain oscillates between its minimum and maximum value, making the vehicle response unpredictable.

The change in piloting technique from the first to the second attempt led to an improvement in tracking error. As shown in Fig. 9, the tracking error for the second attempt by Pilot A at manual steering with AAC active was similar to that of manual steering with AAC disabled, meaning the pilot was able to eliminate or prevent any adverse 
interaction with the adaptive controller. This result is confirmed by observing in Fig. 11 that on the second attempt, the adaptive gain does not oscillate between its limits and remains close to the values from the same test point under autopilot control.

Also of note in Fig. 9, is that the onboard reference model predicted a loss of vehicle due to excessive rigid body loads, i.e., the q-alpha limit, for the autopilot without AAC. Under manual steering, without AAC, the pilot was able to maintain a trajectory that kept the rigid body loads within the acceptable range.

\author{
C. Objective 3: Restrict Unstable Mis-Modeled Parasitic Dynamics to a Bounded Non-Destructive Limit \\ Cycle \\ The project evaluated three Objective 3 test cases with manual steering mode: \\ - Test Case 15: High Gain Controller with Slosh Excitation (Pilot B), \\ - Test Case 16: High Gain Controller with Unstable Flex (Pilot A), and \\ - Test Case 17: High Gain Controller with Rigid Body Instability (Pilot A and Pilot B).
}

\title{
Test Case 15
}

In Test Case 15, the autopilot stabilized the slosh mode both with and without the adaptive controller in the loop. The control commands of Pilot B, without adaptation, however, excited the slosh mode to an instability that caused the system to violate its internal q-alpha limit at approximately the 48-s point of the trajectory. With AAC enabled Pilot B was able to complete the trajectory with only marginally-worse tracking performance than the automatic system, as shown in Fig. 12. The pilot workload was high for the AAC-off case, as would be expected for an unstable system, and is considerably reduced with the activation of the adaptive controller.

Figure 13 shows the pilot command from the AAC-on evaluation of Test Case 15 along with the adaptive gain for both the manual steering and autopilot attempts. In both cases, the slosh dynamics drive the adaptive gain toward its lower limit. Under automatic control, the leakage term periodically raises the gain toward a nominal value of one. Under manual steering, the pilot commands overpower the leakage term and keep the gain at one-half.

\section{Test Case 16}

In Test Case 16, the unstable flex mode violated the SLS vehicle tip deflection limit for all attempts. As shown in Fig. 14, the adaptive controller delayed the limit violation significantly. There was little difference between the autopilot and manual steering with AAC enabled. Without adaptive control, the pilot extended the length of the trajectory by approximately $9 \mathrm{~s}$ as compared with the autopilot case with AAC disengaged, although substantially less than was achieved with AAC enabled.

Activation of the adaptive controller with manual steering mode had little effect on tracking error or pilot workload, as shown in Fig. 15. The relatively low pilot workload with AAC enabled resulted in very little difference in the behavior of the adaptive gain between the autopilot and manual steering mode trajectories in Fig. 16.

\section{Test Case 17}

Test Case 17 was the only Objective 3 manual steering mode evaluation performed by both pilots. Without adaptive control, the unstable rigid body dynamics caused simulated loss of vehicle due to q-alpha violations for both the autopilot and manually-flown trajectories. Tracking error for Pilot A is shown in Fig. 17; the tracking performance of Pilot B was not substantially different. Also shown in Fig. 17 are the pilot workload measures for Test Case 17. Control aggressiveness decreased for each pilot with the activation of the adaptive controller. The measure of duty cycle for Pilot B, however, increased with AAC active. Without AAC a fully-developed PIO occurred, causing Pilot B to apply and hold large, full-scale inputs, reversing direction once every 8 seconds or so. This technique resulted in a low-frequency, high aggressiveness workload measure. The more typical technique used by Pilot B with AAC engaged registered as having a higher duty cycle, but better PIO rating.

Control inputs by both pilots caused the adaptive gain to reach and maintain its minimum value for the duration of the trajectory, whereas under autopilot control the adaptive gain periodically returned toward a nominal value of one before being driven lower again.

\section{Discussion}

\section{A. Objective 1: Minimal Adaptation in the Nominal Case}

An analysis of the manual steering mode for Test Case 0: Nominal Plant and Environment indicates that the pilot and the adaptive controller interact in such a way as to reduce the adaptive gain below its ideal value. Moderately 
aggressive pilot commands are of high enough frequency to be detected by the spectral damper and cause the gain to be lowered. The resulting reduction in forward-loop gain lowers the responsiveness of the vehicle to the pilot's commands, triggering more aggressive inputs. These inputs further reduce the adaptive gain and the result is a tendency by the pilot to over-command the system and drive the adaptive gain to its lower limit. Although the test pilots showed an ability to alter their piloting technique to reduce unwanted interactions, the system showed higher than desired pilot workload and a strong tendency for pilot in the loop oscillations.

These results underscore a key assumption in the design of the AAC: that the presence of parasitic dynamics in the bandwidth of the spectral damper is indicative of a loop gain that is too high and that the correct response is to reduce the adaptive gain. In some cases, the use of manual steering mode violates this assumption. Although none have been identified for the SLS, it is possible that some types of un-modeled dynamics might cause the AAC to adjust its gain in a way that either further excites those dynamics, or fails to adequately attenuate them.

\section{B. Objective 2: Improved Tracking Performance}

The use of manual steering mode in conjunction with AAC for scenarios with reduced control effectiveness, such as Test Cases 5 and 7, consistently resulted in degraded tracking performance when compared to the autopilot with AAC. Without AAC, manual steering mode had about the same error as the autopilot.

The Objective 2 test cases displayed the same tendencies for adverse interactions between the pilot and the adaptive controller that were observed in the Objective 1 results. These interactions varied with pilot technique, but in some cases took the form of large, rapid changes in the adaptive gain, from its minimum allowed value to its maximum value, introducing uncertainty into the control response and flying qualities of the vehicle.

\section{Objective 3: Restrict Unstable Mis-Modeled Parasitic Dynamics to a Bounded Non-Destructive Limit Cycle}

Manual steering mode, due to the higher frequency content of the pilot inputs, helped to lower the adaptive gain for cases in which the design gain was too high, but did not result in improved tracking performance. Certain types of mis-modeled dynamics, such as slosh modes and rigid body modes, may contribute to unstable PIO; the adaptive augmenting controller can help to reduce, but not eliminate, the likelihood of these events. The Objective 3 test cases revealed a tendency for PIO with the manual steering mode.

\section{Summary and Recommendations}

\section{A. Summary}

A prototype version of the SLS flight control software, including its adaptive augmenting control algorithm, was hosted on a piloted aircraft at the AFRC to demonstrate the adaptive controller on a full-scale realistic application in a relevant flight environment. The potential for adverse interactions between the adaptive controller and a proposed manual steering mode was investigated by giving the pilot trajectory deviation cues and pitch rate command authority. Two NASA research pilots flew a total of 25 constant pitch-rate trajectories using a prototype manual steering mode with and without adaptive control, evaluating six different nominal and off-nominal test case scenarios. Pilot comments, including PIO ratings, along with post-flight analysis of aircraft state data and internal controller signals, were used to identify and explain the interactions between the pilot commands and the adaptive algorithm gain.

Although an official SLS manual steering mode design did not exist at the time of these flights, the test results provide insight into potential conflicts between manual control and automatic gain adaptation for such a system. Pilot manual steering did not enhance vehicle performance or robustness beyond what could be achieved by the SLS flight control system with AAC, although under certain failure scenarios the pilot could outperform the SLS control system without adaptive augmenting control.

All of the types of scenarios included in this study showed a tendency for adverse interactions between the pilot and the adaptive augmenting controller under manual steering mode. There is some indication that these interactions can be improved procedurally through alterations in pilot technique, whereby the pilot gain is reduced. It is likely, however, that manual steering mode will be used only in the event of a system failure, when the natural tendency is for pilot gain to increase.

\section{B. Recommendations}

The use of the AAC in conjunction with manual steering mode is not recommended without design changes to mitigate their adverse interaction.

The results of this experiment uncovered scenarios that violate a key assumption in the design of the AAC: that the presence of parasitic dynamics in the bandwidth of the spectral damper is indicative of a loop gain that is too high, 
and that the correct response is to reduce the adaptive gain. Potential solutions to reducing the risk of adverse pilotcontroller interactions take two possible forms: 1) re-design of the AAC algorithm, specifically the spectral damper, to reduce its tendency to identify pilot commands as parasitic dynamics, and 2) modifications that prevent the pilot from commanding dynamics within the identification range of the spectral damper.

Modifications to the structure of the AAC algorithm could potentially alter its performance under autopilot control. The AAC is far along in its development cycle and has been shown to be quite promising. The manual steering mode tested during the LVAC experiment is a prototype design based on assumed requirements, and is therefore much more amenable to changes. Possibilities for preventing the pilot from commanding undesirable dynamics include filtering of the pilot command, and the use of a control stick with an active feel system to increase the force gradient based on the presence of high-frequency inputs. ${ }^{12}$

\section{References}

${ }^{1}$ Wall, J., Orr, J., and VanZwieten, T., "Space Launch System Implementation of Adaptive Augmenting Control," AAS Guidance, Navigation, and Control Conference Proceedings, AAS 14-051, 2014.

${ }^{2}$ Orr, J., and VanZwieten, T., "Robust, Practical Adaptive Control for Launch Vehicles," AIAA Guidance, Navigation, and Control Conference Proceedings, AIAA-2012-4549, 2012.

${ }^{3}$ Brandon, J., Derry, S., Heim, E., Hueschen, R., and Bacon, B., “Ares-I-X Stability and Control Flight Test: Analysis and Plans," AIAA Space 2008 Conference Proceedings, AIAA-2008-7807, 2008.

${ }^{4}$ VanZwieten, T., Gilligan, E., Wall, J., Orr, J., Miller, C., and Hanson, C., "Adaptive Augmenting Control Flight Characterization Experiment on an F/A-18," AAS Guidance, Navigation, and Control Conference Proceedings, AAS 14-052, 2014.

${ }^{5}$ Dennehy, C., VanZwieten, T., Hanson, C., Wall, J., Miller, C., Gilligan, E. and Orr, J., "Flight Testing of the Space Launch System (SLS) Adaptive Augmenting Control (AAC) Algorithm on an F/A-18," NASA/TM-2014-218528, also NESC-RP-1300847.

${ }^{6}$ Thompson, M.O., and Welsh, J.R., "Flight Test Experience with Adaptive Controls Systems," Advanced Control System Concepts, AGARD CP No 58, AGARD Joint Symposium of the Guidance and Control Panel, and the Flight Mechanics Panel of AGARD, pp. 141-147, 1970.

${ }^{7}$ Bosworth, J.T., and Williams-Hayes, P.S., "Flight Test Results from the NF-15B Intelligent Flight Control System Project with Adaptation to a Simulated Stabilator Failure," AIAA-2007-2818.

${ }^{8}$ Hanson, C., Schafer, J., Burken, J.J., Johnson, M. and Nguyen, N., "Handling Qualities Evaluations of Low Complexity Model Reference Adaptive Controllers for Reduced Pitch and Roll Damping Scenarios," AIAA-2011-6607.

${ }^{9}$ McRuer and Krendel, "Mathematical Models of Human Pilot Behavior," AGARDograph No. 188, AGARD-AG-188, 1974.

10 "Flying Qualities of Piloted Aircraft," Mil-Std-1797B, February 15, 2006.

${ }^{11}$ Hanson, C., Schaefer, J., Burken, J. Larson, D., and Johnson, M., "Complexity and Pilot Workload Metrics for the Evaluation of Adaptive Flight Controls on a Full Scale Piloted Aircraft,” NASA/TM-2014-216640.

${ }^{12}$ Klyde, D. and McRuer, D., "Development of Smart-Cue and Smart-Gain Concepts to Alleviate Loss of Control," AIAA-2008-6209, 2008. 


\section{Figures}

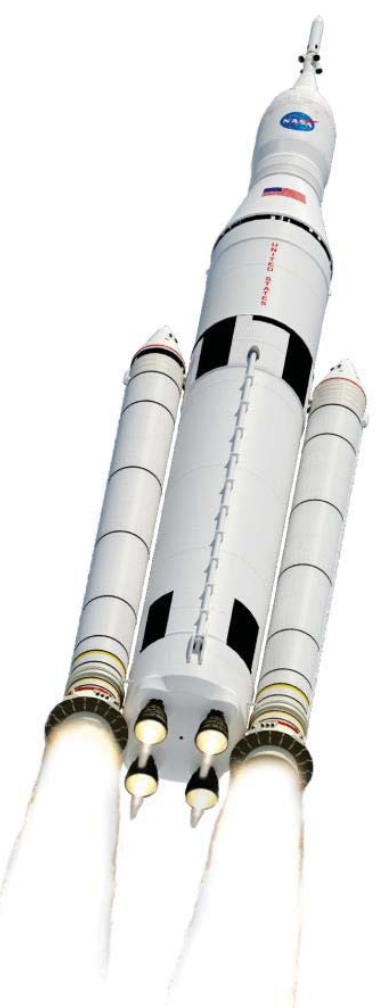

Figure 1. Space Launch System Block I Vehicle.

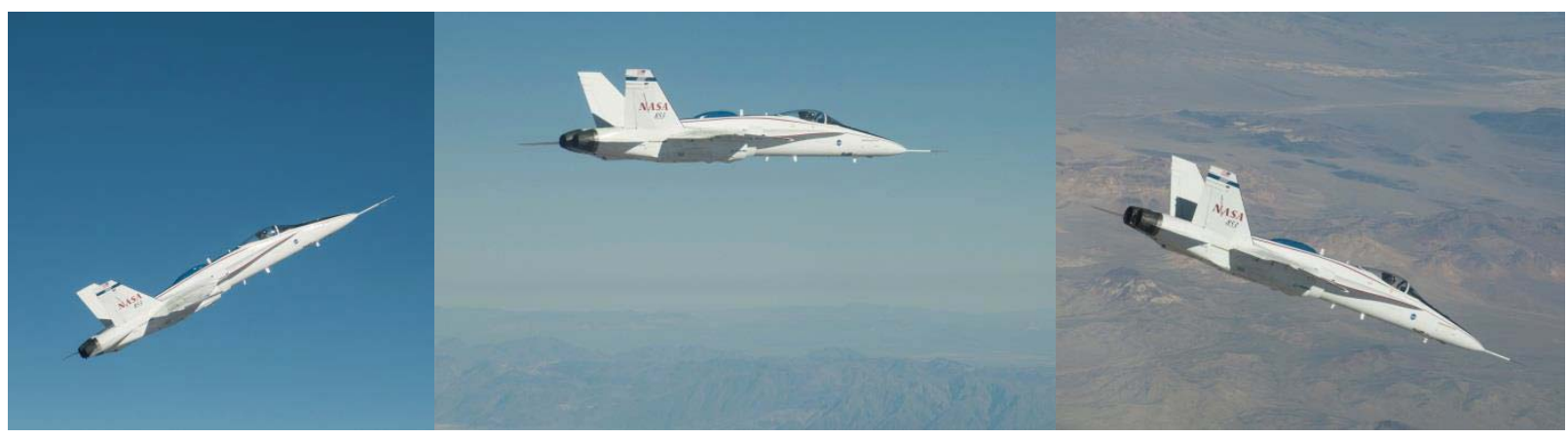

Figure 2. Full-Scale Advanced Systems Testbed (FAST) executing an SLS test trajectory. 


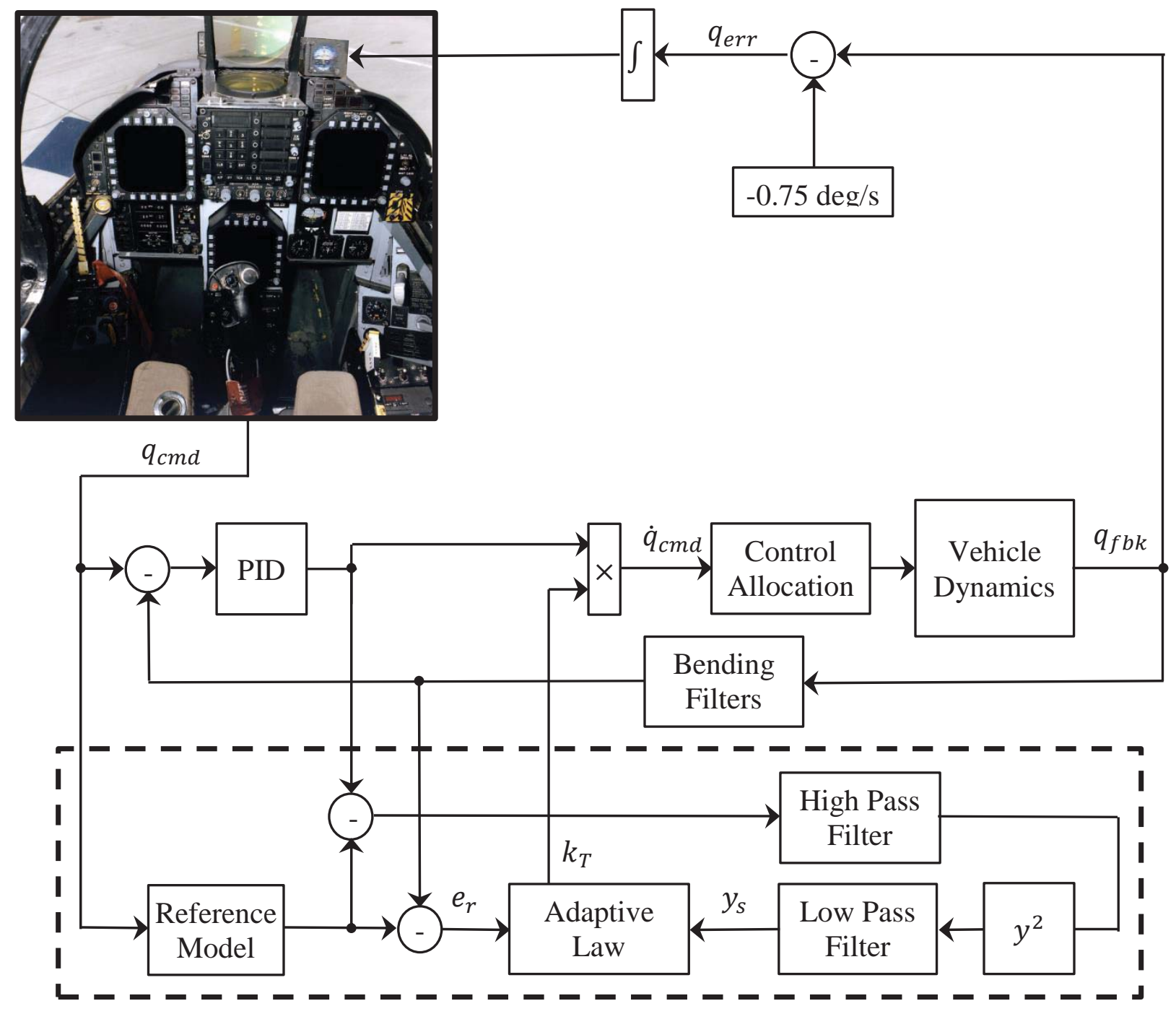

Figure 3. SLS flight control system with Adaptive Augmenting Control and manual steering. 


\section{DECISIONS}

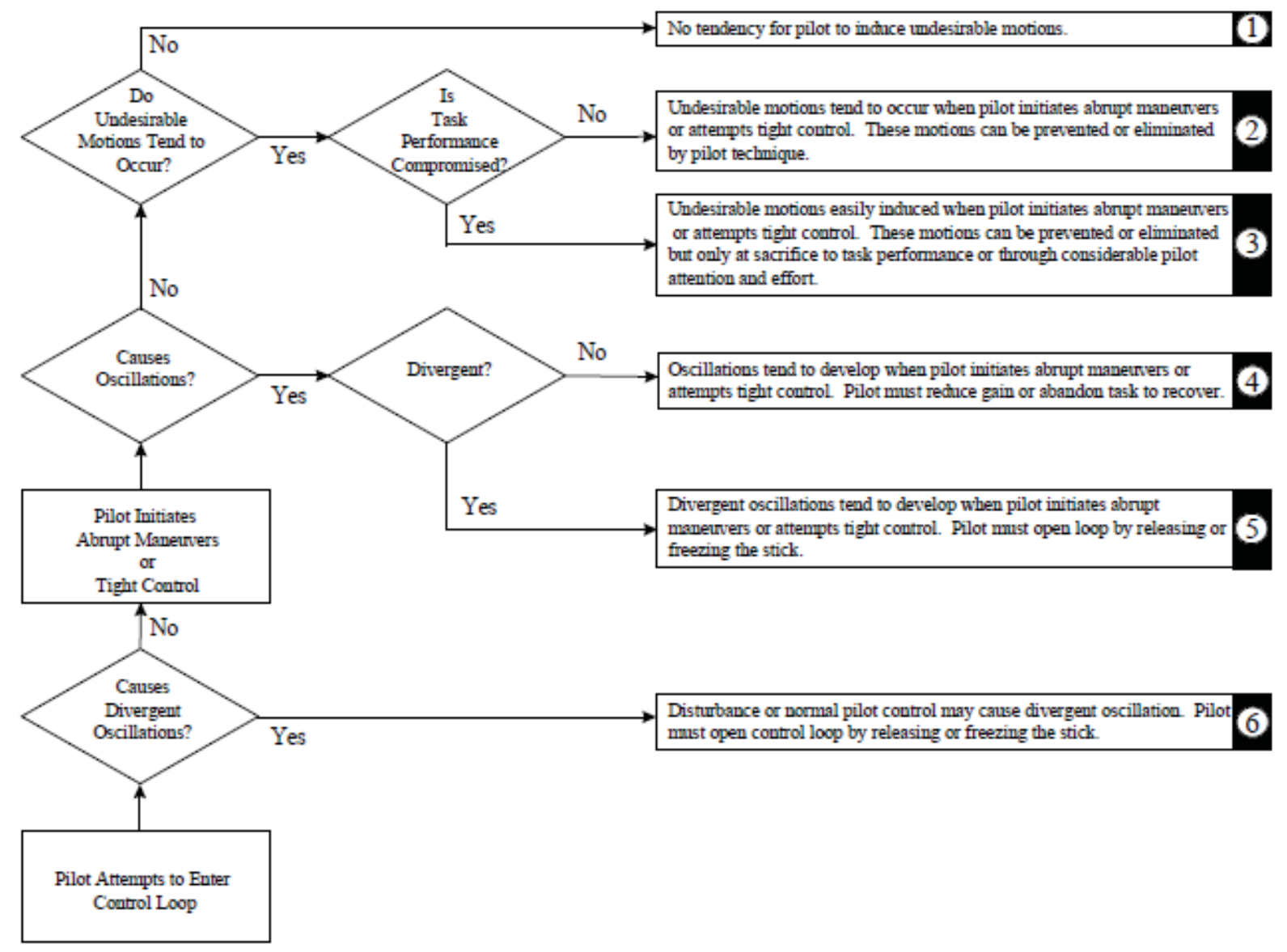

Figure 4. Pilot in the loop oscillation (PIO) rating scale. 


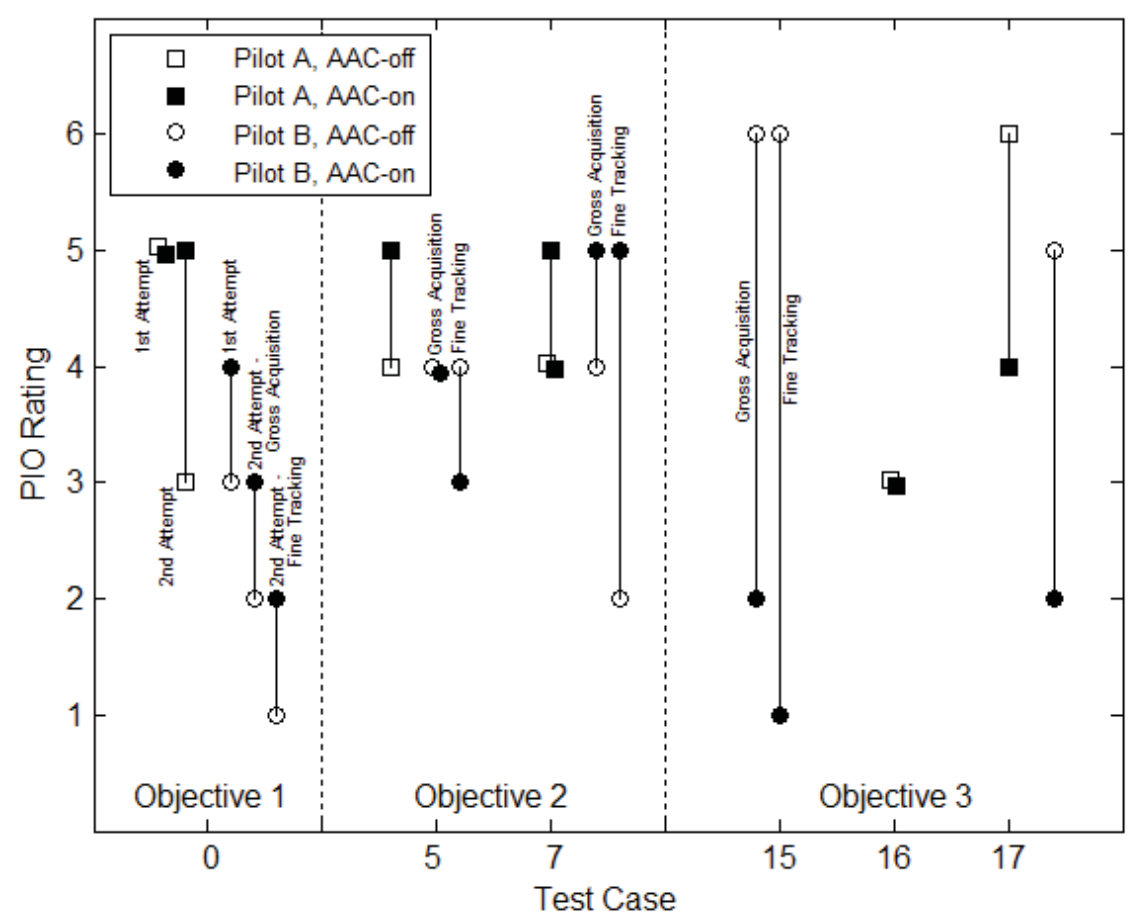

Figure 5. Manual steering mode PIO ratings.

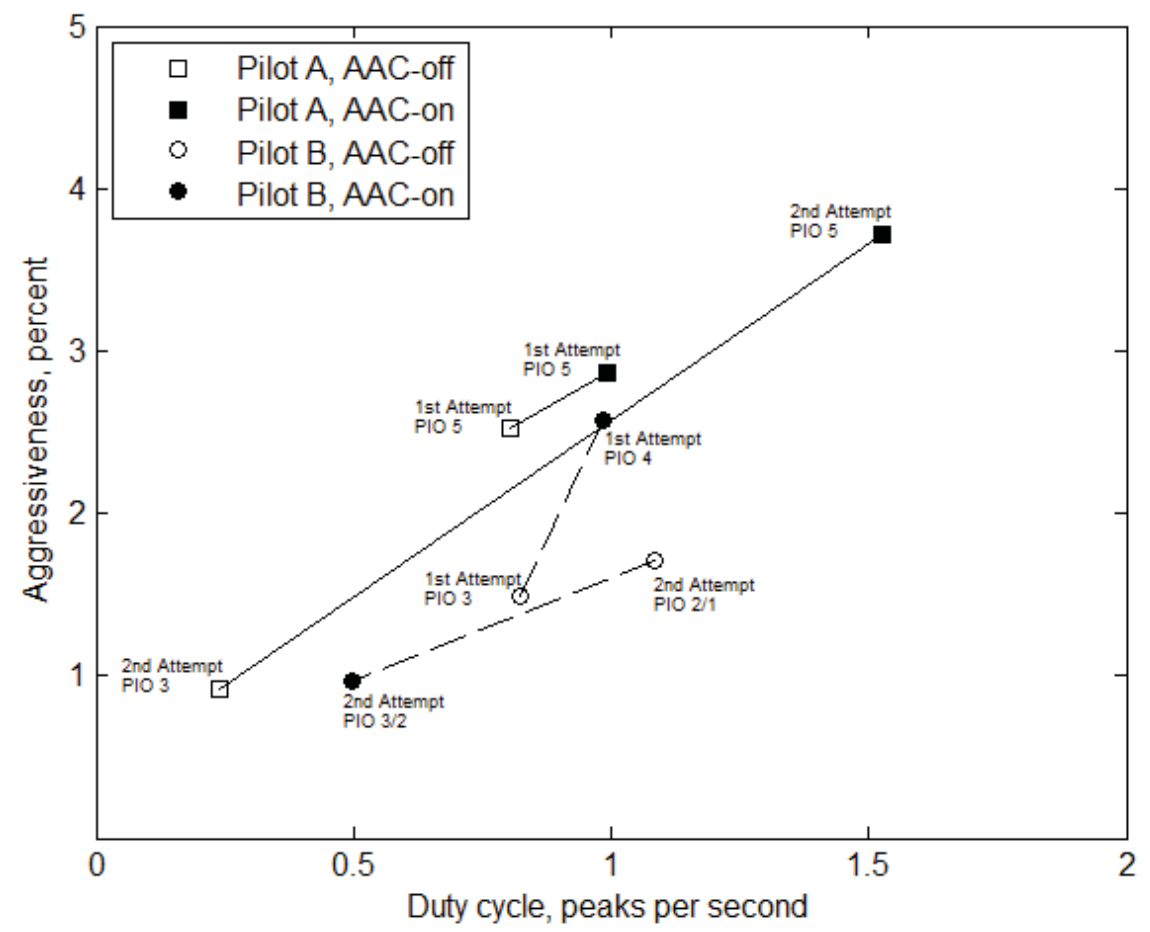

Figure 6. Pilot workload metric results for Case 0: Nominal Plant and Environment. 

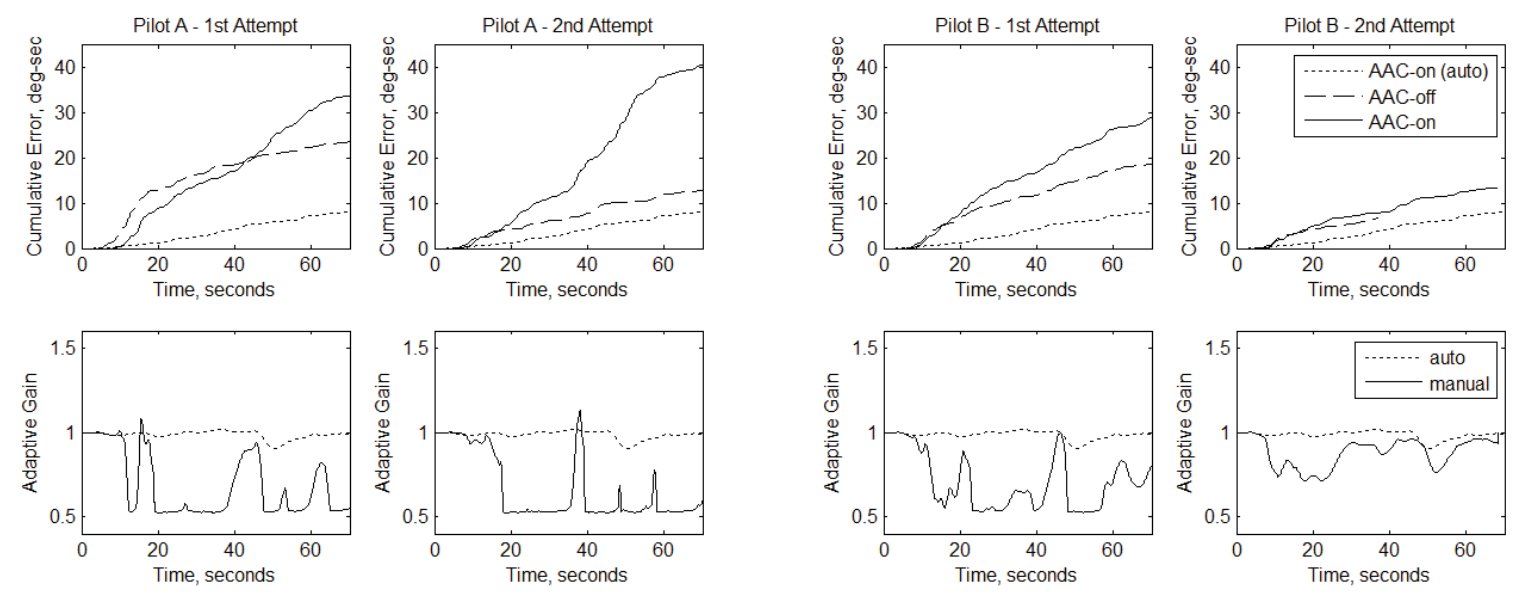

Figure 7. Cumulative tracking error and adaptive gain for Case 0: Nominal Plant and Environment.
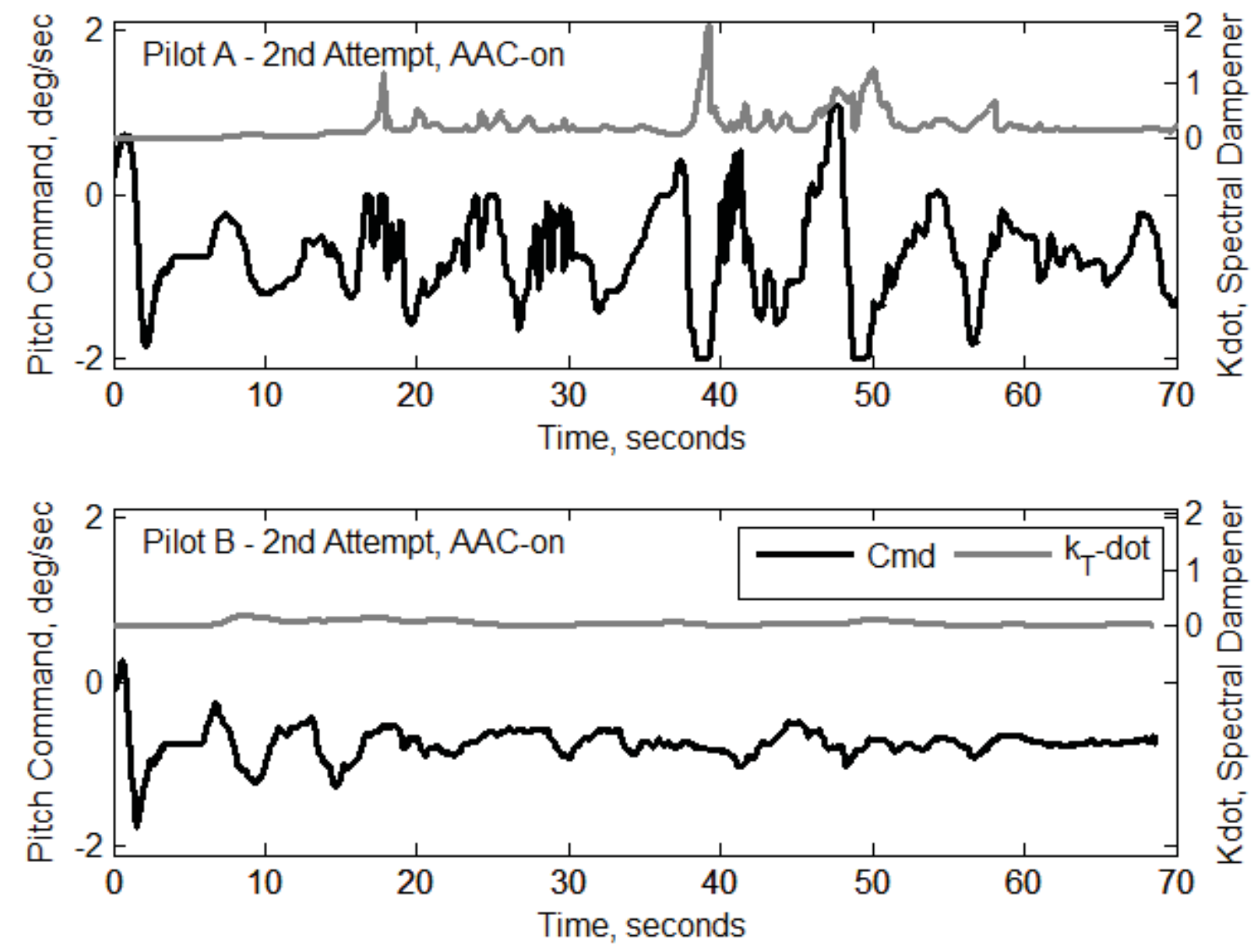

Figure 8. Pitch command and spectral damper contribution to adaptation. 

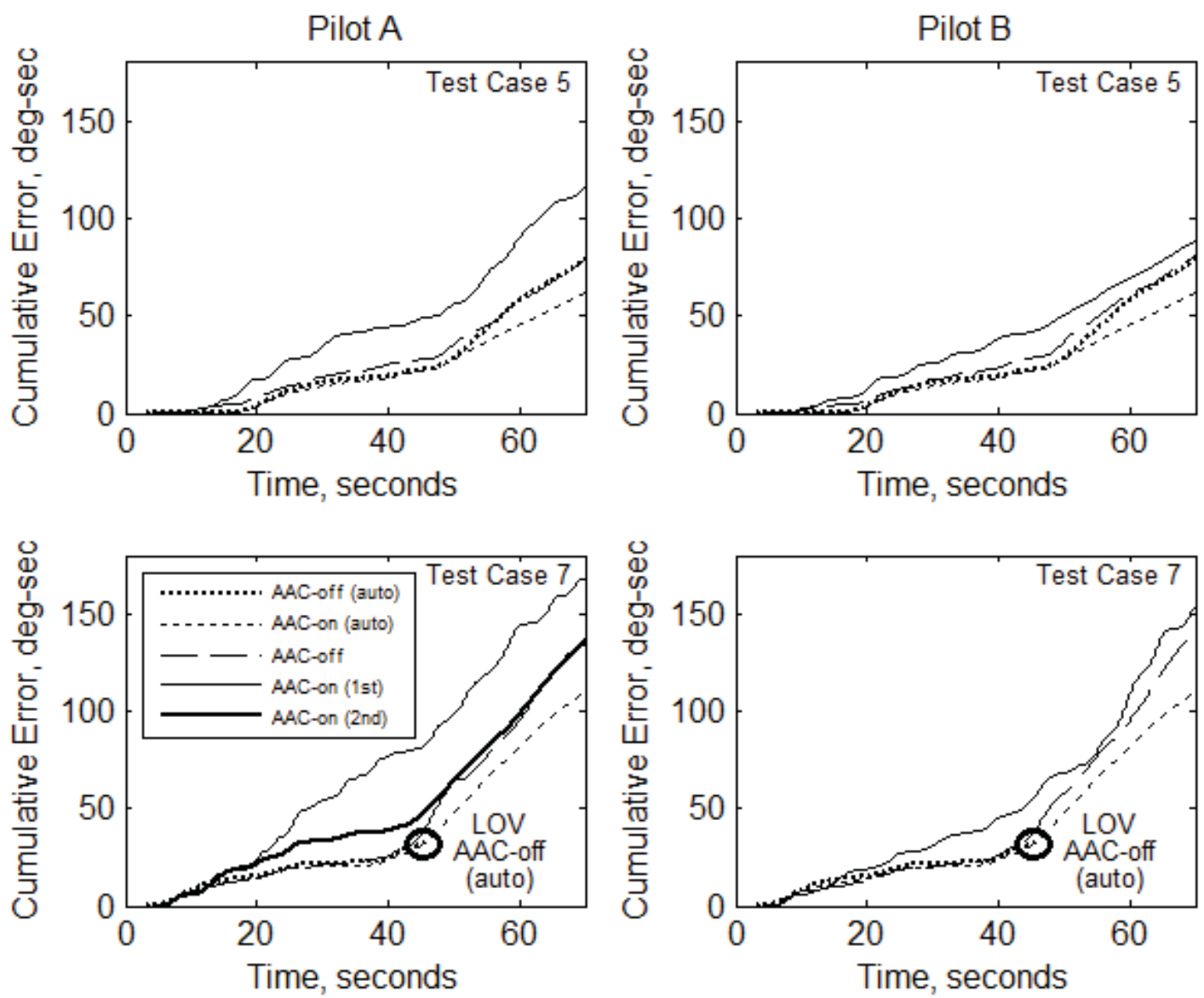

Figure 9. Objective 2 tracking performance results with manual steering mode.
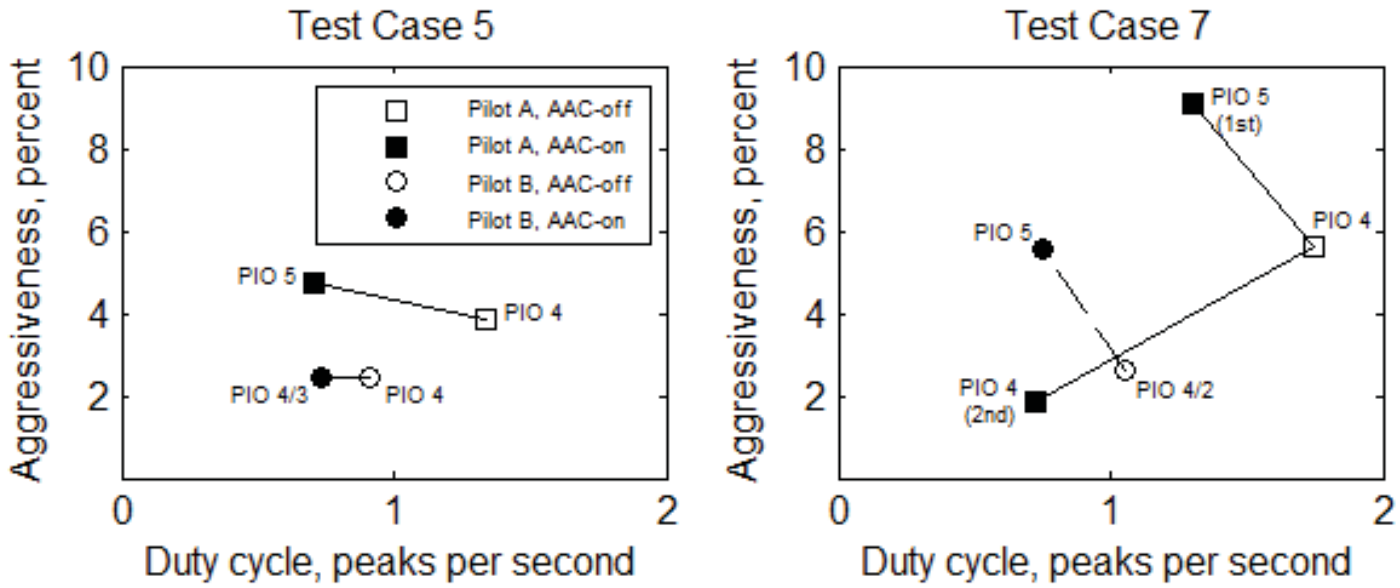

Figure 10. Pilot workload during Objective 2 test case trajectories. 
Pilot A, Test Case 7 - 1st Attempt

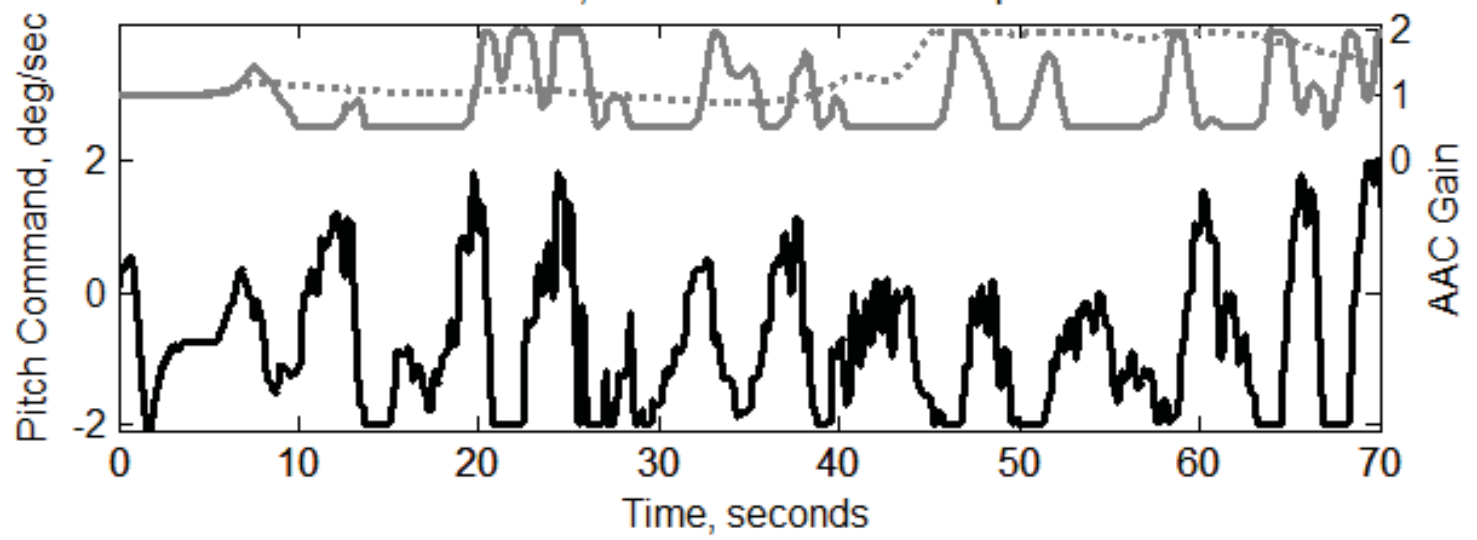

Pilot A, Test Case 7 - 2nd Attempt

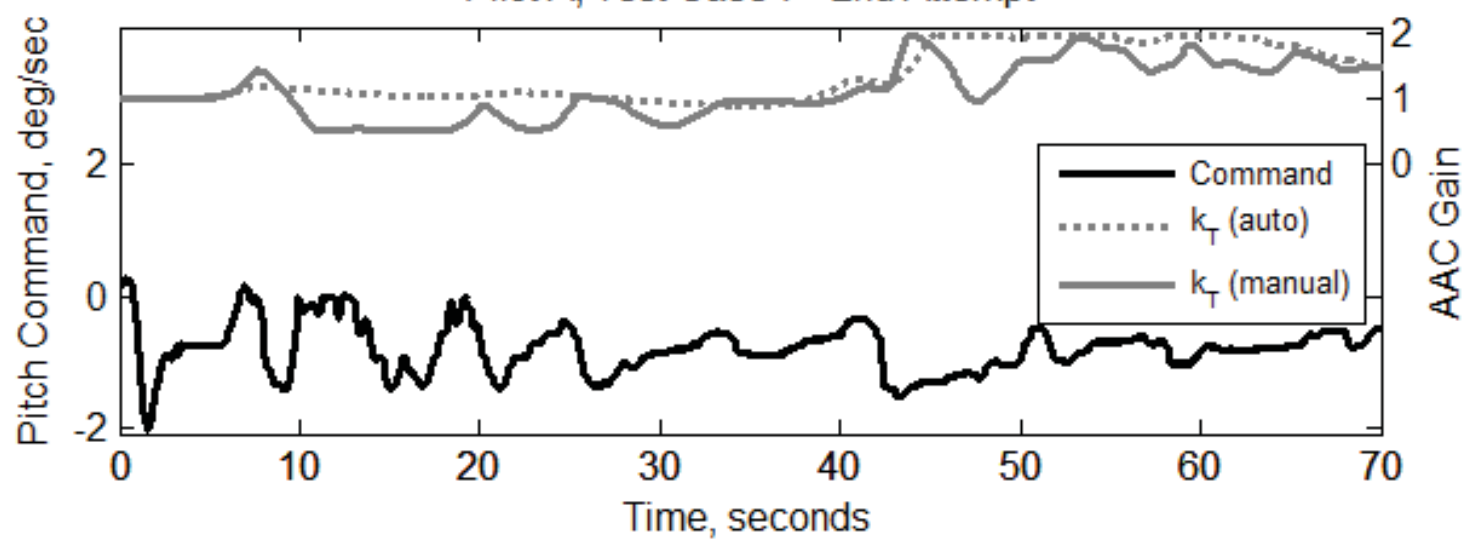

Figure 11. Pitch command and adaptive gain for Objective 2 manual steering mode.
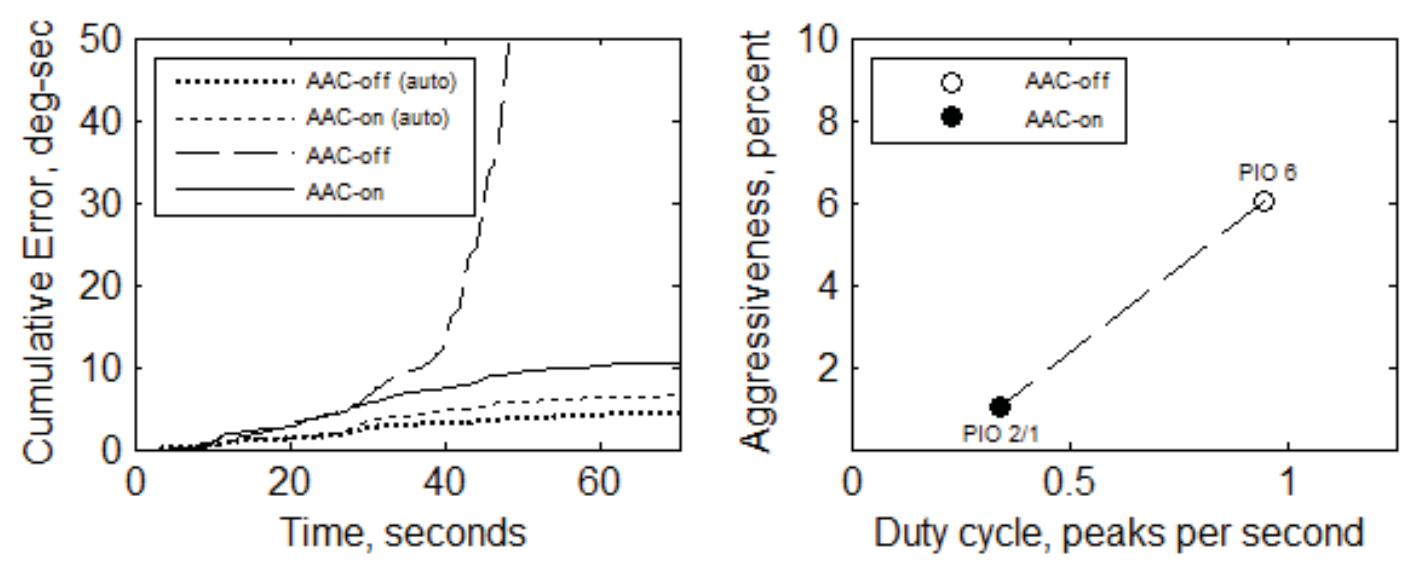

Figure 12. Tracking error and pilot workload, Test Case 15 (Pilot B). 


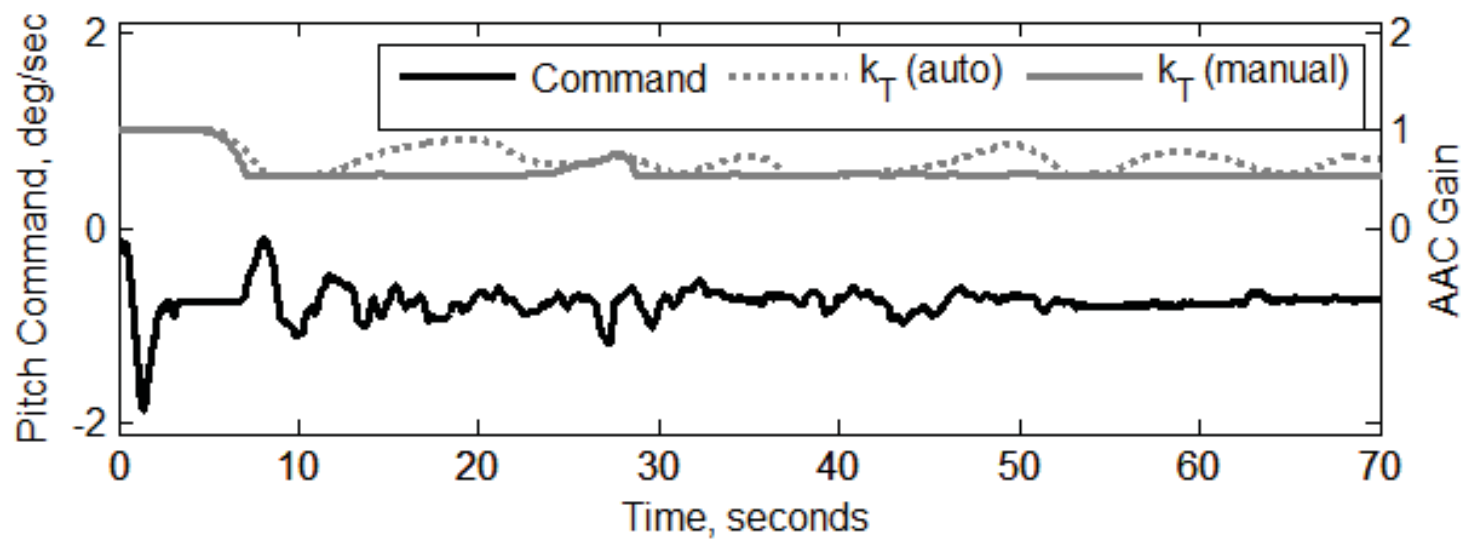

Figure 13. Pilot command and adaptive gain, Test Case 15 (Pilot B).
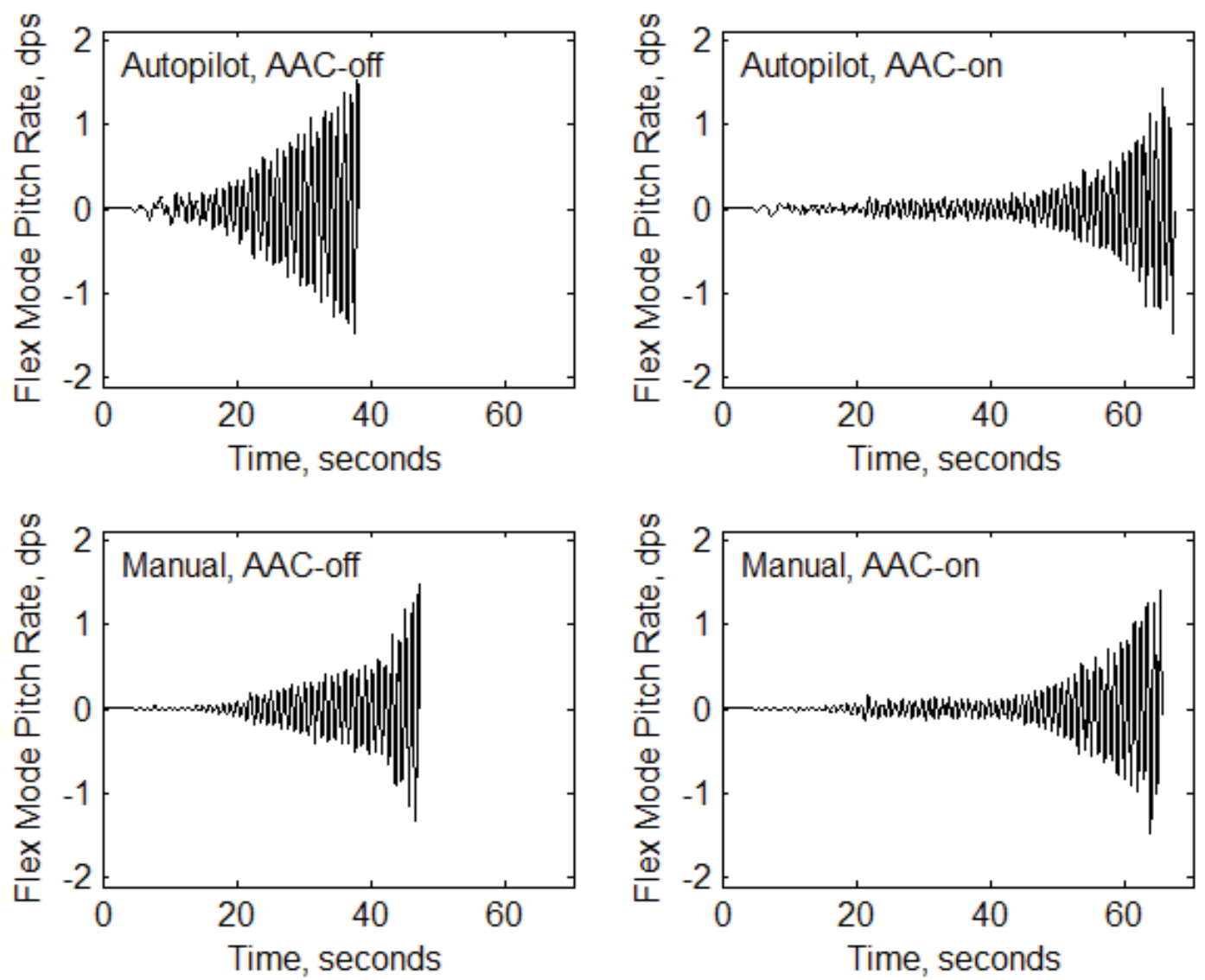

Figure 14. Flex mode pitch rate, Test Case 16 (Pilot A). 

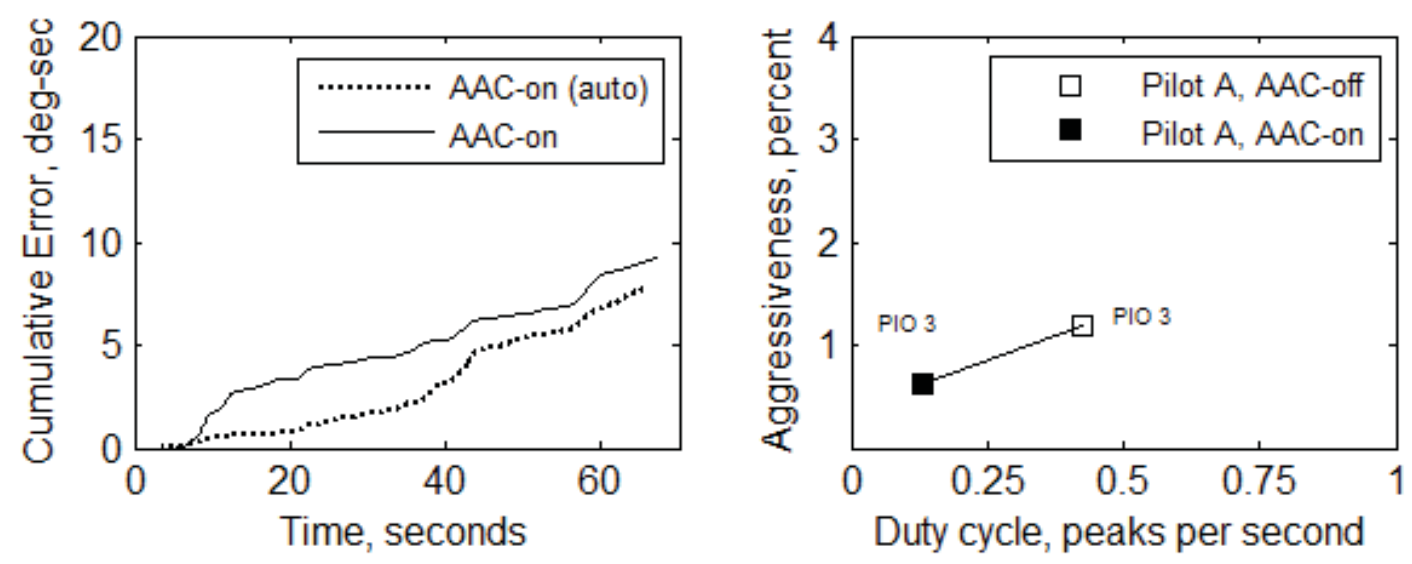

Figure 15. Tracking error and pilot workload, Test Case 16 (Pilot A).

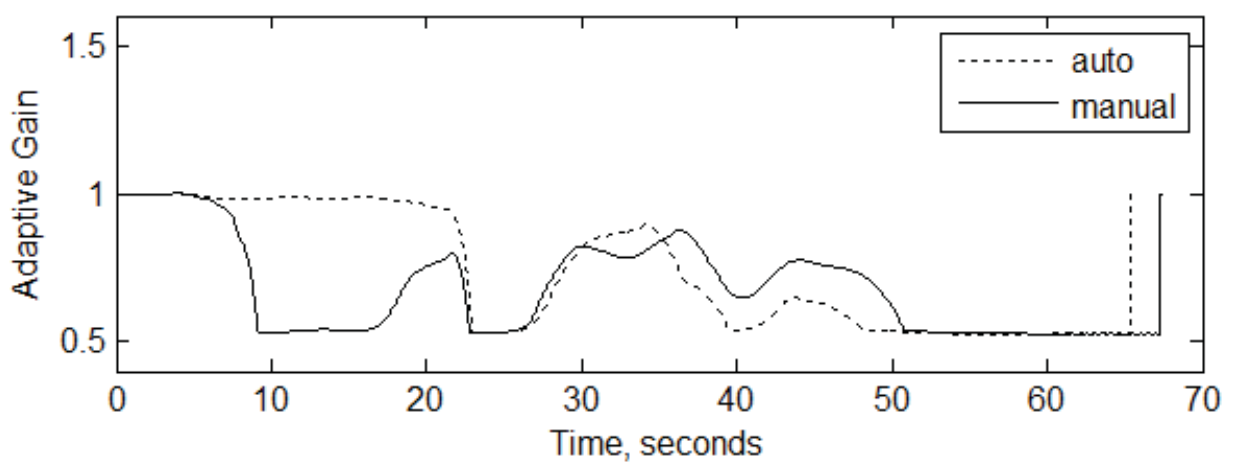

Figure 16. Adaptive gain, Test Case 16 (Pilot A).
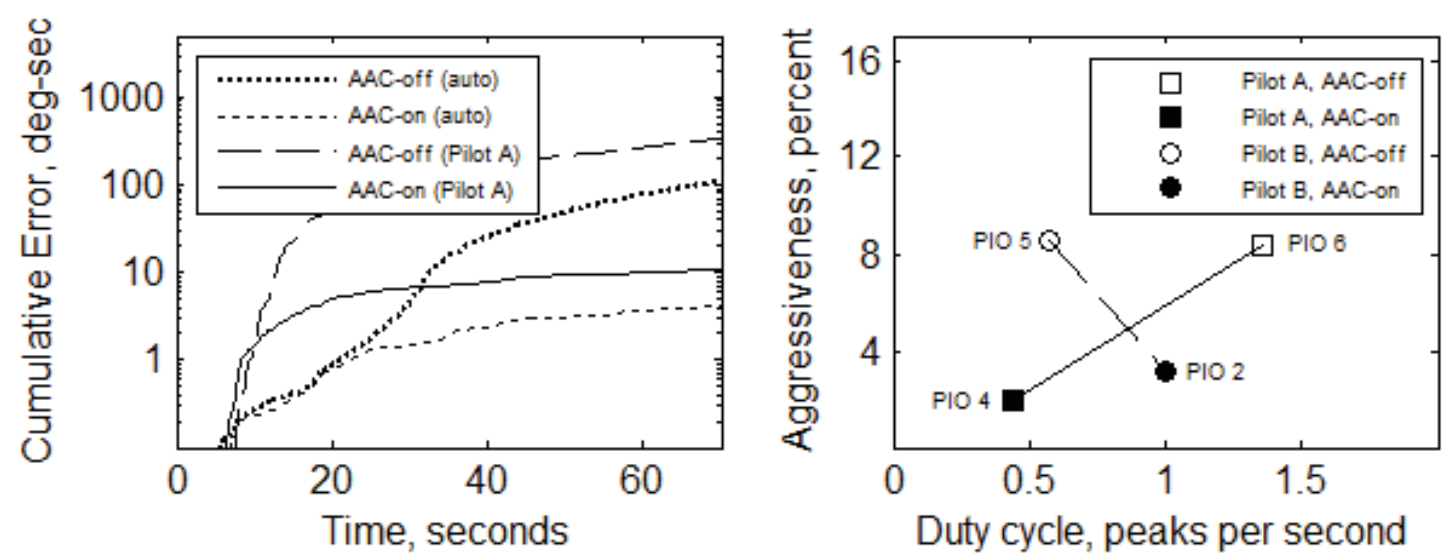

Figure 17. Tracking error and pilot workload, Test Case 17. 